\title{
Does the Market Maker Stabilize the Market?*
}

\author{
Mei Zhu ${ }^{2}$, Carl Chiarella ${ }^{1}$, Xue-Zhong $\mathrm{He}^{1, * *}$ and Duo Wang ${ }^{2}$ \\ ${ }^{1}$ School of Finance and Economics, University of Technology, Sydney \\ PO Box 123, Broadway, NSW 2007, Australia \\ ${ }^{2}$ School of Mathematical Sciences, Peking University \\ Beijing 100871, P. R. China
}

\begin{abstract}
The market maker plays an important role in price formation, but his/her behavior and stabilizing impact on the market are relatively unclear, in particular in speculative markets. This paper develops a financial market model that examines the impact on market stability of the market maker, who acts as both a liquidity provider and an active investor in a market consisting of two types of boundedly rational speculative investors - the fundamentalists and trend followers. We show that the market maker does not necessarily stabilize the market when he/she actively manages the inventory to maximize profits, and that rather the market maker's impact depends on the behavior of the speculators. Numerical simulations show that the model is able to generate outcomes for asset returns and market inventories that are consistent with empirical findings.
\end{abstract}

Keywords: Market maker; inventory; speculators; market stability

\footnotetext{
${ }^{*}$ Current version: March 18, 2009.

This work was initiated while Zhu was visiting the Quantitative Finance Research Centre (QFRC) at the University of Technology, Sydney (UTS), whose hospitality she gratefully acknowledges. The authors would like to thank three anonymous reviewers and Eugene Stanley, the editor, for providing helpful comments. Financial support from the Australian Research Council (ARC) under a Discovery Grant (DP0773776), Paul Woolley Centre for Capital Market Dysfunctionality at the University of Technology, Sydney and National Science Foundation of China Grants (10571003, 10871005) are also gratefully acknowledged.

** Corresponding author. E-mail Address: Tony.He-1@uts.edu.au.
} 


\section{Introduction}

Models of the financial market require some process for the determination of the market price. The two most frequently used mechanisms are the Walrasian auctioneer, widely used in economic theory but which as O'Hara (1995) points out is used in only one market (the market for silver in London), and the market maker mechanism, which is close in spirit to the specialist system used in a number of financial markets.

A market maker can act as a liquidity provider, carrying some inventory in order to accommodate transitory order imbalance, thereby stabilizing markets, as discussed for instance by Wyss (2001). The market maker can also act as an active investor seeking to maximize profits by actively managing his/her inventory. These two different roles of the market maker may often not to be consistent with each other in general. However through the analysis of a rigorous dynamic model with an optimizing market maker, Bradfield (1979) shows that the existence of a target level of inventory that makes the two roles of the market maker consistent with each other. By considering a dynamic model of the market maker incorporating not only inventory but also information effects, Madhavan and Smidt (1993) find that the optimal quotes of the market maker induce mean reversion of his/her inventory towards the target determined by portfolio optimization. The speed of the mean reversion can be different across different markets; it is slow for the New York Stock Exchange (see Madhavan and Smidt (1993)) but relatively fast for the London Stock Exchange (see Hansch, Naik and Viswanathan (1998)).

The market maker inventory can have different impacts on the market price. Empirically, Snell and Tonks (1998) find strong evidence of inventory control effects on price quote revisions on the London Stock Exchange. Madhavan and Sofianos (1998), in a study based on specialist data covering a period of less than two years, find evidence that market makers control their inventories through selectively timing the size and direction of their trades rather than by adjusting their quotes. Recently, using 11 years of New York Stock Exchange specialist data, Hendershott and Seasholes (2007) examine daily inventory and asset price dynamics and find some stylized evidence on inventories, including that the inventories of market makers are negatively correlated with contemporaneous returns and past returns but positively related to subsequent returns.

In general, as indicated in Madhavan and Sofianos (1998), "although specialists play an important role in price formation, we still know relatively little about their behavior and its consequences". Also, in the market inventory literature, much of the attention 
has been focused on the behaviour of the market maker while the behaviour of the other market participants, called "outside customers" by Grossman and Miller (1988), is usually ignored. However, if these are modelled as boundedly rational economic agents, as we do here, then from the recent literature (cited below), we know that the behavior of the outside customers can have a significant impact on the market.

Recent developments in the heterogeneous agent model literature have received much attention as they provide a wide range of explanations of many types of market behaviour and replicate many stylized facts in financial markets that traditional finance has difficulty in explaining. The contributions to this literature are usually either theoretically oriented (as, for example, in Day and Huang (1990), Kirman (1991), Chiarella (1992), Lux (1995), Brock and Hommes $(1997,1998)$ and Chiarella and He (2001, 2002, 2003)) or computationally oriented (as, for example, in Chen and Yeh (2001, 2002), LeBaron (2001, 2002)). We refer the reader to LeBaron (2006), Hommes (2006), Westerhoff (2008), Lux (2009) and Chiarella, Dieci and He (2009) for recent surveys of the developments in this literature. In addition, using tools from statistical physics, recent literature in econophysics has made significant contribution to replicating various stylized facts concerning powerlaw behaviour observed in financial markets, see, for example, Iori (2002), Farmer and Joshi(2002), Cont and Bouchaud (2000), Mantegna and Stanley (2000) and Lux (2009).

Many, though not all, of these models use market maker mechanism to derive the market clearing price and the focus has been mainly on the market clearing role of the market maker. There are few papers in the heterogeneous agent literature examining the impact of investors' positions or the market maker inventories on the market price. Sethi (1996) and Franke and Asada (2008) incorporate the traders' inventory accumulation and cash flow into a Beja and Goldman (1980) framework. Gu (1995) and Day (1997) consider the boundedness of the price adjustment process and the cost of carrying inventories of the market maker. However this literature does not explicitly model the role of the market maker as an active investor. The only exception is the study of Westerhoff (2003) who examines the inventory management of market makers in a heterogeneous foreign exchange market. Through numerical simulations, he shows that certain types of inventory control from the market makers may amplify trading volume and market volatility, and lead to market instability.

This paper is largely motivated by the above literature. The difference between this paper and the current heterogeneous agent model literature is that we model explicitly the role of the market maker as an active investor suggested by the empirical studies of market 
maker inventory. We differ from the traditional market maker inventory literature by incorporating the behaviour of the "outside customers" into the formation of asset prices. We develop a financial market model that examines the impact of a market maker who acts as both a liquidity provider and an active investor on a market consisting of two types of boundedly rational speculative investors, fundamentalists and trend followers. We show that the market maker does not necessarily stabilize the market when actively managing his/her inventory to maximize profit. Different from the traditional inventory literature, the two roles of the market maker can be inconsistent. Depending on the behavior of the fundamentalists and trend followers, the market maker may in fact destabilize the market by actively managing his/her inventory. This paper contributes to an understanding of the stabilizing role of the market maker, which is crucial to practical issues concerning the nature and regulation of financial market design.

The rest of this paper is set out as follows. Section 2 outlines a market maker inventory and boundedly rational heterogeneous agent model. Section 3 analyzes the complicated market behavior in the corresponding deterministic "skeleton" of the model and examines the stabilizing and profit maximizing roles of the market maker. Section 4 provides a statistical analysis of the output of the stochastic version of the model and compares the results to some of the observed empirical facts. Section 5 concludes with some discussion.

\section{The Model}

In this section, we present a heterogeneous agent model of asset price and market maker inventory with a market maker who acts as both a dealer and an active investor. As a dealer, the market maker provides liquidity and carries some inventory in order to accommodate transitory order imbalances, thereby stabilizing markets, see Wyss (2001). As an active investor, the market maker seeks to maintain a long-term target inventory position resulting from his/her optimal portfolio decision to maximize utility or profit (see Bradfield (1979), Madhavan and Smidt (1993) and Hansch, Naik and Viswanathan (1998)). The market price is determined by the market maker in the way described below.

The Market - Consider a market with two assets, one risk-free and one risky (such as stock), a market maker and a number of outside customers, so called by Grossman and Miller (1988), consisting of $H$ types of $N(N \geq H)$ investors with different expectations on the future payoffs of the risky asset. Let the gross return of the risk-free asset be 
$R=1+r$, where $r$ is the risk-free rate per trading period. Let $P_{t}$ and $D_{t}$ be the price and dividend of the risky asset in trading period $t$ defined as $[t, t+1)$. The dividend process $D_{t}$ is assumed to be an i.i.d. stochastic process with mean $\bar{D}$, and all investors have an unbiased estimate of $D_{t}$.

Portfolio Optimization and Market Aggregation-Our framework is that of a repeatedly optimizing one-period dynamical model. Following the standard decision making under uncertainty theory, we assume that all investors choose their optimal portfolio of the risk free and risky assets so as to maximize their expected utility. More precisely, let $z_{s}$ denote the total supply of the risky asset in quantity terms, which is assumed to be fixed. Let $R_{t+1}=P_{t+1}+D_{t+1}-R P_{t}$ be the excess return of the risky asset over the trading period $t+1$. Let $z_{h, t}$ be the number of shares held by a typical investor- $h$ in trading period $t$, based on the current market price $P_{t}$ and historical prices, and $W_{h, t}$ be his/her wealth in trading period $t$. Given the market price $P_{t}$ and the wealth $W_{h, t}$ of a typical investor $h$ in trading period $t$, the portfolio wealth for the typical investor in trading period $t+1$ is given by

$$
W_{h, t+1}=R\left(W_{h, t}-P_{t} z_{h, t}\right)+\left(P_{t+1}+D_{t+1}\right) z_{h, t}=R W_{h, t}+R_{t+1} z_{h, t} .
$$

Let $E_{h, t}\left(X_{t+1}\right)$ and $V_{h, t}\left(X_{t+1}\right)$ be the beliefs or forecasts of type $h$ investors about the conditional expectation and conditional variance of quantity $X$ over the trading period $t+1$, based upon their information up to trading period $t$. Then it follows from (2.1) that $E_{h, t}\left(W_{h, t+1}\right)=R W_{h, t}+E_{h, t}\left(R_{t+1}\right) z_{h, t}$ and $V_{h, t}\left(W_{h, t+1}\right)=V_{h, t}\left(R_{t+1}\right)\left(z_{h, t}\right)^{2}$. For traders of type $h$, assume that their utility functions are given by $U_{h}(W)=-e^{-a_{h} W}$ with a constant absolute risk aversion coefficient $a_{h}$. Assume that $R_{t+1}$ is conditionally normally distributed based on the information up to time $t$. By maximizing their expected utility of wealth in trading period $t+1$, the optimal holding for the risky asset, denoted $z_{h, t}^{*}$, of type $h$ investors in trading period $t$ is determined as

$$
z_{h, t}^{*}=\frac{E_{h, t}\left(R_{t+1}\right)}{a_{h} V_{h, t}\left(R_{t+1}\right)} .
$$

Therefore, the aggregate holding (or demand) of the risky asset from the $H$ types of investors over the trading period $t$ is given by

$$
z_{t}^{*}:=\sum_{h=1}^{H} n_{h} z_{h, t}^{*}=\sum_{h=1}^{H} n_{h} \frac{E_{h, t}\left(R_{t+1}\right)}{a_{h} V_{h, t}\left(R_{t+1}\right)}
$$


where $n_{h}$ denotes the number of type- $h$ investors over all trading periods and hence $\sum_{h=1}^{H} n_{h}=N$.

Market Maker - The market maker is assumed to act as both a dealer and an active investor. As an active investor, the empirical evidence suggests that the market maker manages his/her portfolio so as to maintain a long-term target inventory position $I^{d}$ in the risky asset which is consistent with his/her portfolio objective. More precisely, let $I_{t}$ be the inventory of the market maker in trading period $t$. Following Madhavan and Smidt (1993), the desired inventory position of the market maker at the beginning of trading period $t+1, I_{t+1}^{d}$, is assumed to be a weighted average of the current inventory in trading period $t$ and the long-run desired inventory level $I^{d}$, so that

$$
I_{t+1}^{d}=I_{t}+\alpha\left(I^{d}-I_{t}\right), \quad \alpha \in[0,1]
$$

where the coefficient $\alpha$ measures the speed of inventory adjustment of the market maker, which may be related to the risk aversion of the market maker (see Bradfield (1979)). When $\alpha=0, I_{t+1}^{d}=I_{t}$, meaning that the market maker does not adjust his/her position actively to the target inventory level and hence the role of the market maker as an active investor disappears. When $\alpha=1, I_{t+1}^{d}=I^{d}$, implying that the market maker would like to adjust immediately to his/her target inventory in the next trading period. The meanreverting process of the inventory documented in the inventory empirical literature is in general characterized by $\alpha \in(0,1)$. That is, the market maker wants to adjust his/her current inventory position in trading period $t$ to the target level for trading period $t+1$ in a smooth fashion. In this way, when $I_{t}<I^{d}\left(I_{t}>I^{d}\right)$, the market maker smoothly increases (decreases) the current inventory position towards the target inventory.

On the other hand, as a dealer, the market maker has an obligation to provide liquidity and to stabilize the market by charging commissions. More precisely, the market maker inventory $I_{t+1}$ of the risky asset at the beginning of trading period $t+1$ is given by

$$
I_{t+1}=z_{s}-z_{t}^{*}
$$

where $z_{t}^{*}$ is the aggregate optimal demand of all $H$ types of investors in trading period $t$. That is, (2.5) states that the inventory of the market maker in period $t+1$ is equal to the total supply of the risky asset minus the investors optimal holding of the asset. Taking into account the desired inventory $I_{t+1}^{d}$ of the market maker as an active investor, 
the market excess demand for the risky asset in trading period $t+1$ can be written as

$$
I_{t+1}^{d}-I_{t+1}=z_{t}^{*}-\left(z_{s}-I_{t+1}^{d}\right)
$$

where $z_{s}-I_{t+1}^{d}$ is the desired supply of the market maker to the outside investors. In trading period $t+1$, the market maker clears the market by taking an opposite long (short) position when $I_{t+1}^{d}-I_{t+1}<0(>0)$. In this paper we assume that the market maker is able to take whatever position is required to clear the market and hence short selling is permitted.

Under the market maker scenario, the market maker sets the market price of the risky asset according to the market excess demand. In trading period $t$, the market maker announces the price of the stock $P_{t}$ based on information of the previous trading period $t-1$. For the outside investors, based on the information available up to trading period $t$ and their heterogeneous beliefs about the price $P_{t+1}$ and dividend $D_{t+1}$, their aggregate optimal demand $z_{t}^{*}$ for trading period $t$ is determined by equation (2.3). In order to reach their optimal holding for the current trading period $t$, the outside investors submit market orders $^{1}$ in the current trading period, and hence buy or sell the shares of the stock at price $P_{t}$. The market maker then takes the opposite position to clear the market at the same price $P_{t}$. As an active investor, the desired inventory $I_{t+1}^{d}$ of the market maker for the trading period $t+1$ is given by equation (2.4). By taking into account the fixed supply $z_{s}$, the excess demand of the risky asset in the market is given by equation (2.6). If the excess demand $z_{t}^{*}-\left(z_{s}-I_{t+1}^{d}\right)$ is positive (negative), the market maker increases (decreases) the market price from $P_{t}$ to $P_{t+1}$ in trading period $t+1$. The outside traders adjust their holdings to their optimal demands in trading period $t+1$ by submitting market orders at price $P_{t+1}$ and the market maker takes an off setting position to clear the market at the same price $P_{t+1}$. The market then moves to the next trading period $t+2$ and so on. Figure 1 displays a time line that lays out the sequence of actions in the market, where $M M$ indicates an action by the market maker and $O$ an action by the outside agents.

In order to avoid negative prices, we adopt the rule of relative price adjustment used by Zheng (2007). Such a rule posits that the market maker adjusts the price so that the return is an increasing function of the market excess demand, and the function is zero when the excess demand is zero and bounded when the excess demand becomes infinite. A typical example would be

$$
P_{t+1}=P_{t}\left[1+\rho \tanh \left(\mu\left[z_{t}^{*}-\left(z_{s}-I_{t+1}^{d}\right)\right]\right)\right]
$$

\footnotetext{
${ }^{1}$ Note that here we consider a simple model that ignores limit orders.
} 
where $\mu>0$ determines the magnitude of sensitivity of the market maker to the excess demand and $\rho \in(0,1]$ determines the upper and lower bounds of the adjustment when the excess demand is large.

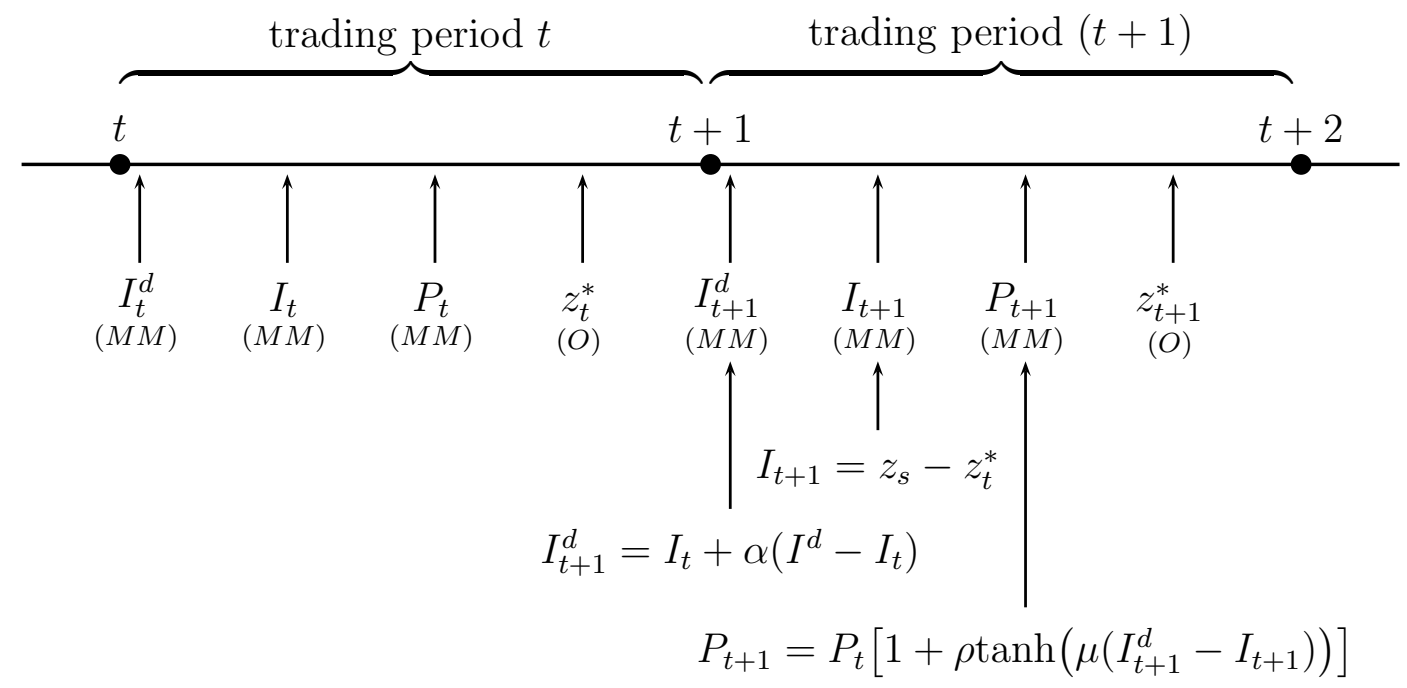

Figure 1: The sequencing of events in the market. Here $M M$ indicates an action by the market maker and $O$ an action by the outside agents.

Rational Fundamental Equilibrium Price - In order to obtain a benchmark fundamental price, we first consider the case when all investors are homogeneous and have rational expectation. Following the framework of Brock and Hommes $(1997,1998)$, we thus assume $E_{h, t}\left(R_{t+1}\right)=E_{t}\left(R_{t+1}\right), V_{h, t}\left(R_{t+1}\right)=V_{t}\left(R_{t+1}\right)=\sigma^{2}$, and $a_{h}=a$ for $h=1, \cdots, H$. Thus the total demand of outside investors in the trading period $t$ is given by

$$
z_{t}^{*}=N \frac{E_{t}\left(R_{t+1}\right)}{a V_{t}\left(R_{t+1}\right)}=N \frac{E_{t}\left(P_{t+1}\right)+\bar{D}-R P_{t}}{a \sigma^{2}} .
$$

In a rational expectation equilibrium, the long-run fundamental equilibrium price $P_{t}^{*}$ of the risky asset is determined when the demand equals the supply, so that

$$
N \frac{E_{t}\left(P_{t+1}^{*}\right)+\bar{D}-R P_{t}^{*}}{a \sigma^{2}}+I^{d}=z_{s},
$$

leading to

$$
P_{t}^{*}=\frac{1}{R}\left[E_{t}\left(P_{t+1}^{*}\right)+\bar{D}-s\right],
$$


where $s=a \sigma^{2}\left(z_{s}-I^{d}\right) / N$. Then, under the assumption of the 'no bubble' condition $\lim _{k \rightarrow \infty} \frac{E_{t}\left(P_{t+k}^{*}\right)}{R^{k}}=0$, the fundamental price is given by

$$
P_{t}^{*}=P^{*}=\frac{\bar{D}-s}{r}
$$

A Market Fraction Model-To model the impact of the outside customers in the market, we follow $\mathrm{He}$ and $\mathrm{Li}(2007,2008)$ and consider the simplest market fraction heterogeneous agent model with two types of beliefs - these of the fundamentalists and trend followers, which is a simple version of the adaptive heterogeneous agent model introduced in Brock and Hommes (1998). As in Brock and Hommes (1998) and Hommes, Huang and Wang (2005), we assume that $a_{1}=a_{2}=a$ and $V_{1, t}\left(R_{t+1}\right)=V_{2, t}\left(R_{t+1}\right)=\sigma^{2}$, but that fundamentalists and trend followers have different beliefs in the first moment of excess return $R_{t+1}$.

Fundamentalists - Following He and Li (2008), we assume that fundamentalists have superior information on the fundamental value, $P_{t}^{*}$, of the risky asset. They believe that the stock price may be driven away from the fundamental value in the short-run, but will eventually converge back to the fundamental value in the long-run. This fundamental price may reflect not only the future dividends but also other information such as earnings and $\mathrm{P} / \mathrm{E}$ ratio. In this paper, we assume that the fundamental price follows a random walk process starting at the constant fundamental price $P^{*}$, so that

$$
P_{t+1}^{*}=P_{t}^{*}\left[1+\widetilde{\epsilon}_{t+1}\right], \quad \widetilde{\epsilon}_{t} \sim \mathcal{N}\left(0, \sigma_{\epsilon}^{2}\right), \quad \sigma_{\epsilon} \geq 0, \quad P_{0}^{*}=P^{*}=\frac{\bar{D}-s}{r}>0,
$$

where $\widetilde{\epsilon}_{t}$ is i.i.d. normal random variable with zero mean and a constant variance $\sigma_{\epsilon}^{2}$. This specification ensures that high kurtosis, fat tails and power-law behaviour in asset returns are not brought about by the exogenous news arrival process reflected in the fundamental value. Hence the emergence of any correlation pattern of the asset return and market maker inventory and the autocorrelation patterns in the inventories, returns, absolute and squared returns discussed in Section 4 would be driven by the trading process itself, rather than the process for the fundamental value. We assume that the fundamentalists calculate the conditional mean of the future price according to

$$
E_{1, t}\left(P_{t+1}\right)=P_{t}+k^{\prime}\left(E_{t}\left(P_{t+1}^{*}\right)-P_{t}\right)=P_{t}+k^{\prime}\left(P_{t}^{*}-P_{t}\right)
$$


where the parameter $k^{\prime} \in[0,1]$ is the speed of price adjustment of the fundamentalists towards the fundamental value, which also measures the fundamentalists level of confidence in the fundamental value. In particular, for $k^{\prime}=1$, the fundamentalists are fully confident about the fundamental value and adjust their expected price next period instantaneously to the expected fundamental value. For $k^{\prime}=0$, the fundamentalists become naive traders. Accordingly, the optimal demand of the fundamentalists for the risky asset is given by

$$
z_{1, t}^{*}=\frac{E_{1, t}\left(R_{t+1}\right)}{a V_{1, t}\left(R_{t+1}\right)}=\frac{E_{1, t}\left(P_{t+1}-P_{t}\right)+\bar{D}-r P_{t}}{a V_{1, t}\left(R_{t+1}\right)}=\frac{k^{\prime}\left(P_{t}^{*}-P_{t}\right)+\bar{D}-r P_{t}}{a \sigma^{2}} .
$$

Trend Followers - Unlike the fundamentalists, trend followers are technical traders who believe that the future price change can be predicted from various patterns or trends generated from past prices. We also assume that the trend followers expect that the price trend will continue when the deviation of the current market price from the latest price is small but become more cautious the further the deviation goes beyond some threshold value. The expectation of the trend followers is based on the same idea as in Chiarella, Dieci and Gardini (2002), but the idea of cautiousness is similar to that used by Westerhoff (2004) and Zheng (2007), however here it is applied in a different way. More precisely the conditionally expected price change of the trend followers is assumed to evolve over time based on the past price change by

$$
E_{2, t}\left(P_{t+1}-P_{t}\right)=a^{\prime}\left(P_{t}-P_{t-1}\right) e^{-c\left(P_{t}-P_{t-1}\right)^{2}+b\left(P_{t}-P_{t-1}\right)}
$$

where parameter $a^{\prime}>0$ denotes the intensity of extrapolation of the trend followers, the parameter $b \in \mathbb{R}$ captures the asymmetry of beliefs about the condition of the market ( $b>0$ for a bull market and $b<0$ for a bear market), and the parameter $c>0$ indicates the intensity of asymmetric reaction of the trend followers' extrapolation on the past price change. The expression (2.12) indicates that if $P_{t}-P_{t-1}>0$ the trend followers expect an increase in price if the past price change $P_{t}-P_{t-1}$ is smaller than some threshold value (calculated below), but beyond it they believe that they are in a price bubble that will soon burst and so expect lower future price trends. A similar argument applies when $P_{t}-P_{t-1}<0$. Of course, they react symmetrically to the price changes if $b=0$.

Let $y(x)=a x e^{-c x^{2}+b x}$ with $x=P_{t}-P_{t-1}$ and $y$ denoting $E_{2, t}\left(P_{t+1}-P_{t}\right)$, then the threshold values are given by $x_{ \pm}^{*}=\left[b \pm \sqrt{b^{2}+8 c}\right] /(4 c)$ and $y_{\max }=y\left(x_{+}^{*}\right), y_{\min }=y\left(x_{-}^{*}\right)$. For $a^{\prime}=1, b=-1, c=2$, the function $y(x)$ is plotted in Fig. 2. In this case, $x_{+}^{*}=$ $0.39, x_{-}^{*}=-0.64$ and $y_{\max }=0.1948, y_{\min }=-0.535$. In this example, $\left|x_{-}^{*}\right|>\left|x_{+}^{*}\right|$, 
the trend followers are more bearish than bullish and they become cautious only when negative price changes are larger than positive price changes.

Thus using (2.12) the optimal demand for the trend followers on the risky asset in trading period $t$ is given by

$$
\begin{aligned}
z_{2, t}^{*} & =\frac{E_{2, t}\left(R_{t+1}\right)}{a V_{2, t}\left(R_{t+1}\right)}=\frac{E_{2, t}\left(P_{t+1}-P_{t}\right)+\bar{D}-r P_{t}}{a V_{2, t}\left(R_{t+1}\right)} \\
& =\frac{a^{\prime}\left(P_{t}-P_{t-1}\right) e^{-c\left(P_{t}-P_{t-1}\right)^{2}+b\left(P_{t}-P_{t-1}\right)}+\bar{D}-r P_{t}}{a \sigma^{2}} .
\end{aligned}
$$

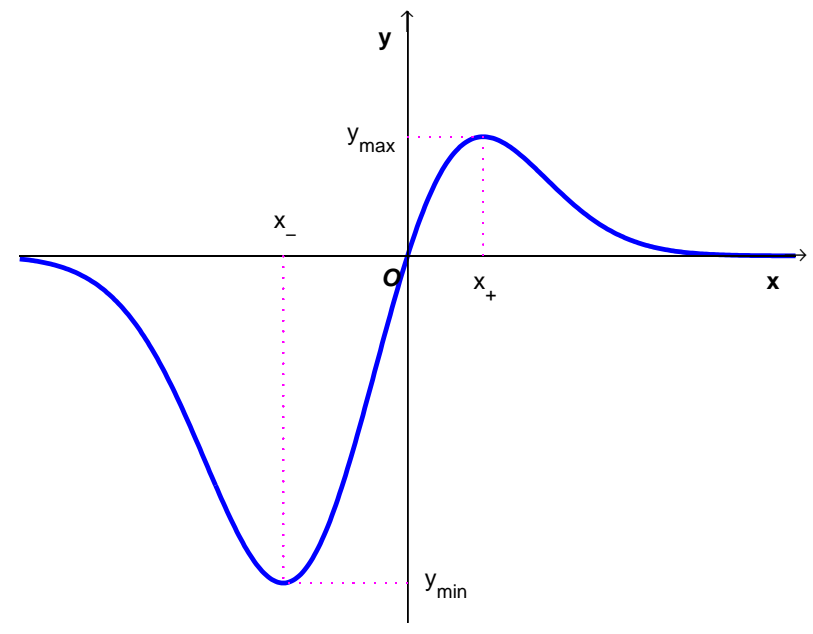

Figure 2: The expectation function $y=x e^{-2 x^{2}-x}$ of the trend followers. In the example illustrated here the trend followers are more bearish than bullish. Note that $x=P_{t}-P_{t-1}$ and $y=E_{2, t}\left(P_{t+1}-P_{t}\right)$.

Noise Traders - In addition, in order to capture unexpected market news or other noise traders in the market, we introduce a noisy demand term $\widetilde{\delta}_{t}$ which is an i.i.d. normally distributed random variable with $\widetilde{\delta}_{t} \sim \mathcal{N}\left(0, \sigma_{\delta}^{2}\right)$.

The Price and Inventory Dynamics - Hence based on (2.5), the aggregate demand of outside traders in trading period $t$ and the market maker inventory at the beginning of trading period $t+1$ are given by

$$
\begin{aligned}
z_{t}^{*} & =n_{1} z_{1, t}^{*}+n_{2} z_{2, t}^{*}+\widetilde{\delta}_{t+1}, \\
I_{t+1} & =z_{s}-z_{t}^{*}=z_{s}-n_{1} z_{1, t}^{*}-n_{2} z_{2, t}^{*}-\widetilde{\delta}_{t+1} .
\end{aligned}
$$


Substituting (2.2), (2.4) and (2.14) into (2.7), we find that the price and the inventory dynamics under a market maker scenario are determined by the random discrete dynamical system

$$
\left\{\begin{array}{l}
P_{t+1}=P_{t}\left[1+\rho \tanh \left(\mu\left(n_{1} z_{1, t}^{*}+n_{2} z_{2, t}^{*}+I_{t}+\alpha\left(I^{d}-I_{t}\right)-z_{s}+\widetilde{\delta}_{t+1}\right)\right)\right] \\
I_{t+1}=z_{s}-n_{1} z_{1, t}^{*}-n_{2} z_{2, t}^{*}-\widetilde{\delta}_{t+1} \\
P_{t+1}^{*}=P_{t}^{*}\left(1+\widetilde{\epsilon}_{t+1}\right)
\end{array}\right.
$$

where $z_{1, t}^{*}$ and $z_{2, t}^{*}$ are defined by (2.11) and (2.13), respectively.

In the following sections we undertake theoretical analysis and numerical simulations of the model (2.16). For convenience of analysis and notation, we let $n_{1}:=\frac{n_{1}}{N}, n_{2}:=\frac{n_{2}}{N}, I_{t}:=$

$\frac{I_{t}}{N}, I_{d}:=\frac{I_{d}}{N}, z_{s}:=\frac{z_{s}}{N}, \widetilde{\delta}_{t+1}=\frac{\widetilde{\delta}_{t+1}}{N}, \mu:=\mu N$. Thus $n_{1}+n_{2}=1$ and $P^{*}=\frac{\bar{D}-a \sigma^{2}\left(z_{s}-I^{d}\right)}{r}$. In order to understand the impact of the market maker on market stability, in Section 3, we first analyze the dynamics of the deterministic version of the model (2.16) by assuming $\sigma_{\delta}=\sigma_{\epsilon}=0$, and hence the fundamental price is constant. Using numerical simulation, we also analyze in Section 3 the impact of the two noise processes on the deterministic dynamics and the deviation of the market price from the fundamental price. In particular, we examine the roles of the market maker in stabilizing the market and maximizing profit.

\section{The Stabilizing Role of the Market Maker}

In order to obtain some theoretical understanding of the stochastic model, we first examine the underlying deterministic model. By assuming $\sigma_{\delta}=\sigma_{\epsilon}=0$, we have $P_{t}^{*}=P^{*}$, $\widetilde{\delta}_{t}=0$, then the stochastic model (2.16) becomes the deterministic model

$$
\left\{\begin{array}{l}
P_{t+1}=P_{t}\left[1+\rho \tanh \left(\mu\left(n_{1} z_{1, t}^{*}+n_{2} z_{2, t}^{*}+I_{t}+\alpha\left(I^{d}-I_{t}\right)-z_{s}\right)\right)\right] \\
I_{t+1}=z_{s}-n_{1} z_{1, t}^{*}-n_{2} z_{2, t}^{*}
\end{array}\right.
$$

where $z_{1, t}^{*}$ and $z_{2, t}^{*}$ are defined by (2.11) and (2.13), respectively. Let $\bar{a}=\frac{\mu \rho P^{*} a^{\prime} n_{2}}{a \sigma^{2}}$ and $\bar{k}=\frac{\mu \rho P^{*}\left(k^{\prime} n_{1}+r\right)}{a \sigma^{2}}$, which are respectively the extrapolation rate of the trend followers and the speed of mean reversion of the fundamentalists, adjusted by population proportion, variance, risk aversion and the market maker's speed of price adjustment. Our main results can be stated as follows.

Proposition 1 For $0<\alpha<1$, the model (3.1) has a fundamental equilibrium steady state $\left(\bar{P}_{1}, \bar{I}_{1}\right)=\left(P^{*}, I^{d}\right)$ and a non-fundamental equilibrium $\left(\bar{P}_{2}, \bar{I}_{2}\right)=\left(0, z_{s}-\frac{k^{\prime} n_{1} P^{*}+\bar{D}}{a \sigma^{2}}\right)$. 
The non-fundamental equilibrium is always unstable, while the fundamental equilibrium $\left(\bar{P}_{1}, \bar{I}_{1}\right)$ is locally asymptotically stable if the parameters $(\alpha, \bar{a}, \bar{k})$ lie inside the region $D$, where

$$
\begin{aligned}
& D(\alpha, \bar{a}, \bar{k})=\{(\alpha, \bar{a}, \bar{k}): 0<\bar{a} \leq 1, \quad 0<\bar{k}<\frac{2}{2-\alpha}+2 \bar{a} \\
&\text { or } \left.1<\bar{a}<\frac{1}{1-\alpha}, \quad 0<\bar{k}<\min \left\{\frac{2}{2-\alpha}+2 \bar{a}, \frac{\bar{a}-1-\alpha(1-\alpha) \bar{a}^{2}}{(1-\alpha)(1-\bar{a})}\right\}\right\} .
\end{aligned}
$$

In addition,

- for $0<\bar{a} \leq 1$, the fundamental equilibrium becomes unstable through the flip bifurcation boundary defined by $\bar{k}=\frac{2}{2-\alpha}+2 \bar{a}$;

- for $1<\bar{a}<\frac{1}{1-\alpha}$, the fundamental equilibrium becomes unstable through either the flip bifurcation boundary defined by $\bar{k}=\frac{2}{2-\alpha}+2 \bar{a}$ or the Neimark-Sacker bifurcation boundary defined by $\bar{k}=\frac{\bar{a}-1-\alpha(1-\alpha) \bar{a}^{2}}{(1-\alpha)(1-\bar{a})}$.

Proposition 1 gives the explicit conditions for the local stability of the fundamental equilibrium and the induced bifurcation in terms of the three key parameters $\alpha, \bar{a}$ and $\bar{k}$, which characterise the activities of the market maker, the trend follows and the fundamentalists, respectively. ${ }^{2}$ The proof of the proposition and a more systematic theoretical analysis of the different roles of various parameters and the complexity of the dynamics due to different types of bifurcations can be found in Chiarella, He, Wang and Zhu (2008). The parameter $\alpha$ corresponds to the speed of mean-reversion of the inventory to the target long-term inventory level, measuring the activity of the market maker as an active investor. It is determined by the portfolio decision of the market maker in general. As a dealer, the market maker is expected to provide liquidity to the market and hence to stabilize the market. In the following discussion, we first focus on the stabilizing role of the market maker who acts as an active investor, that is the stabilizing effect of the parameter $\alpha$. We will see that, depending on the activity of the fundamentalists and

\footnotetext{
${ }^{2}$ The parameters $\mu$ and $\rho$ play the following roles. Since $\bar{a}$ and $\bar{k}$ are both monotone increasing functions of $\mu$ and $\rho$, Proposition 1 implies that for given $\alpha,(\bar{a}, \bar{k})$ cross the flip or Neimark-Sacker boundary and move in to the unstable region as $\mu$ or $\rho$ increases. That is, the fundamental equilibrium $\left(P^{*}, I^{d}\right)$ becomes unstable through the flip or the Neimark-Sacker bifurcation as $\mu$ or $\rho$ increases. These results are also consistent with those on the speed of price adjustments of the market maker in the previous literature (see for example, Chiarella and He (2003)) and the motivation of setting the limit of price change (hope to stabilize the market).
} 


\begin{tabular}{|c|c|c|c|c|c|c|c|c|c|c|c|}
\hline$P^{*}$ & $\rho$ & $\mu$ & $n_{1}$ & $n_{2}$ & $z_{s}$ & $I^{d}$ & $r$ & $\bar{D}$ & $a \sigma^{2}$ & $\mathrm{~b}$ & $\mathrm{c}$ \\
\hline 10 & 0.1 & 2 & 0.5 & 0.5 & 1 & 0.1 & 0.0002 & 0.902 & 1 & -1 & 0.1 \\
\hline
\end{tabular}

Table 1: The common set of parameter values used in the numerical analysis.

trend followers, an increase of $\alpha$ can destabilize the market, pushing the market price away from the fundamental price and generating excess volatility.

In order to see the impact of the speed of the inventory adjustment of the market maker $\alpha$ on the stability of the fundamental equilibrium, we use the results of Proposition 1 to plot in Fig. 3 cross-sectional diagrams of the local stability regions $D$ in $(\alpha, \bar{k})$ parameter space for three different values of $\bar{a}$.

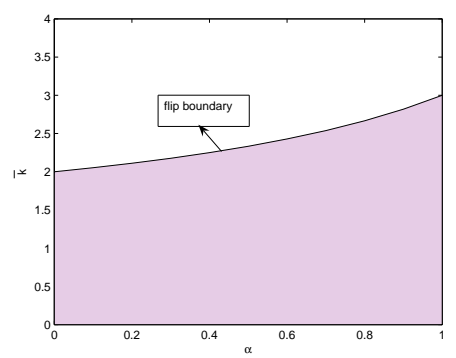

(a) $\bar{a}=0.5$

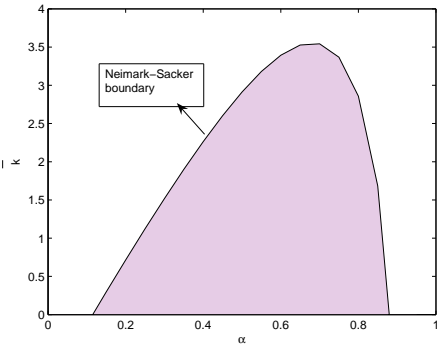

(b) $\bar{a}=1.13$

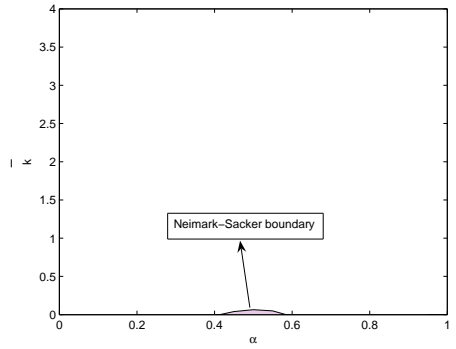

(c) $\bar{a}=1.7$

Figure 3: The local stability regions (shaded) and bifurcation boundaries in $(\alpha, \bar{k})$ parameter space with different values of $\bar{a}$ : (a) $\bar{a}=0.5$, instability occurs via a flip bifurcation, (b) $\bar{a}=1.13$, instability occurs via Neimark-Sacker bifurcations, and (c) $\bar{a}=1.7$, the Neimark-Sacker bifurcation boundary diminishes and the stability region disappears.

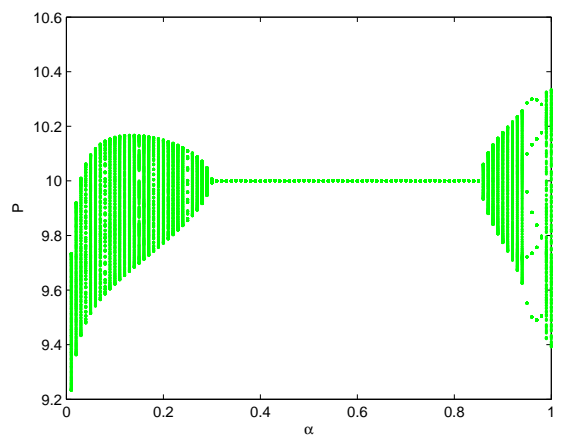

Figure 4: The bifurcation diagram of the market price $P_{t}$ with respect to parameter $\alpha$, here $\bar{a}=1.13, \bar{k}=1.5$.

The Stabilizing Role of the Market Maker-Fig. 3 shows different stability regions with respect to $\alpha$, depending upon the relative strength of the reaction of the 
fundamentalists and trend followers. For fixed $\bar{a}=0.5$, Fig. 3 (a) demonstrates the stabilizing role of the market maker by showing how the stability region of $\bar{k}$ becomes larger as $\alpha$ increases. As $\bar{a}$ increases to 1.13, Fig. 3 (b) shows that the stabilizing role of the market maker is effective only when $\alpha$ is neither too low nor too high. This implies that, when the trend followers are relatively more active, the market maker destabilizes the market when his/her speed of mean-reversion to the target inventory is either too slow or too fast. This is verified by the bifurcation diagram with respect to the parameter $\alpha$ in Fig. $4^{3}$. When the trend followers become even more active, Fig. 3 (c) shows that the market becomes unstable almost independently of $\alpha$.

\begin{tabular}{|c|c|c|c|c|c|}
\hline$\alpha$ & 0.1 & 0.3 & 0.5 & 0.7 & 0.9 \\
\hline$(\bar{a}, \bar{k})=(0.5,2.5)$ & 0.4247 & 0.3085 & 0.2148 & 0 & 0 \\
\hline$(\bar{a}, \bar{k})=(1.13,1.5)$ & 0.2007 & 0 & 0 & 0 & 0.1452 \\
\hline
\end{tabular}

Table 2: Deviations of the market prices from the fundamental price $S D_{P P^{*}}$ of the deterministic model in the two cases $(\bar{a}, \bar{k})=(0.5,2.5)$ and $(1.13,1.5)$ for five values of $\alpha$.

In order to provide a quantitative description on the effects of the market maker on the market stability, in Table 2, we show the standard deviations of the market prices from the fundamental prices $S D_{P P^{*}}$ over the time period ${ }^{4} T$ for changing $\alpha$ in the two cases of $(\bar{a}, \bar{k})$ for the deterministic model, where $S D_{P P^{*}}$ is defined by

$$
S D_{P P^{*}}=\sqrt{\frac{1}{T} \sum_{t=1}^{T}\left(P_{t}-P_{t}^{*}\right)^{2}} .
$$

For the first case $(\bar{a}, \bar{k})=(0.5,2.5)$, it reflects the fact that the trend followers are less active and the fundamentalists are relatively more active. For the deterministic model, from Table 2, we see that the deviation of the market prices from the fundamental prices $S D_{P P^{*}}$ diminishes as $\alpha$ increases. This result is consistent with the stabilizing role as $\alpha$ increases illustrated in Fig. 3 (a). In the second case $(\bar{a}, \bar{k})=(1.13,1.5)$, the trend followers become more active but the fundamentalists are relatively less active. In this

\footnotetext{
${ }^{3}$ All the numerical results except for those on profits in this section are based on the common set of parameters in Table 1.

${ }^{4}$ Given that we are mainly concerned about the long run asymptotic properties, we take $T=10,000$ by dropping the first 1,000 of 11,000 observations in our statistical analysis of market stability and the profit of the market maker.
} 
case, from Table 2, we see that either large or small $\alpha$ leads to instability of the market, which is consistent with Fig. 3 (b) and Fig. 4.

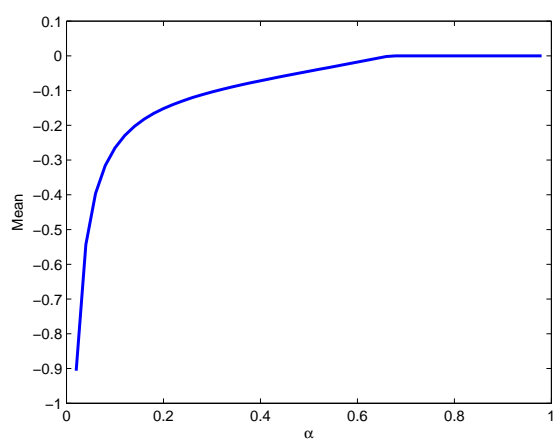

(a) Mean $\left(\sigma_{\epsilon}, \sigma_{\delta}\right)=(0,0)$

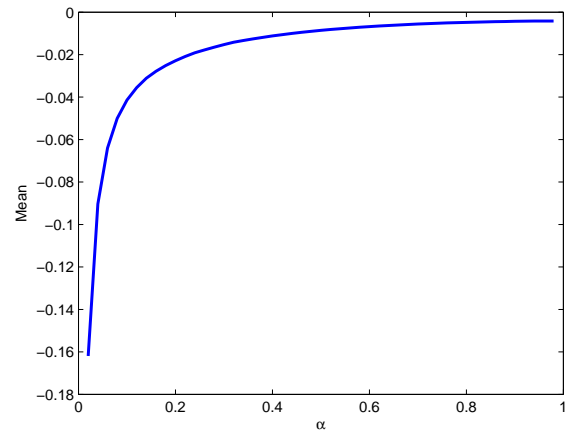

(c) Mean $\left(\sigma_{\epsilon}, \sigma_{\delta}\right)=(0.01,0.005)$

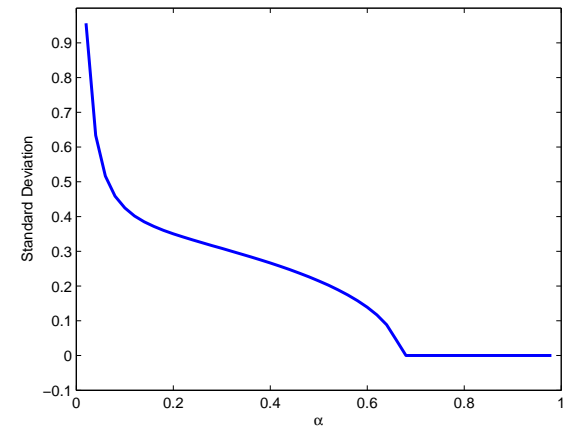

(b) $S D_{P P^{*}}\left(\sigma_{\epsilon}, \sigma_{\delta}\right)=(0,0)$

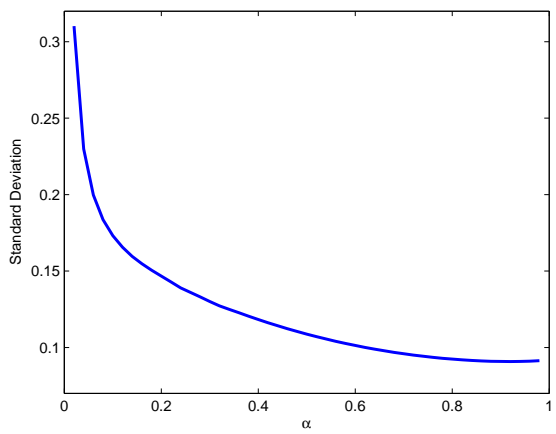

(d) $S D_{P P^{*}}\left(\sigma_{\epsilon}, \sigma_{\delta}\right)=(0.01,0.005)$

Figure 5: Mean and standard deviation of the $P_{t}-P_{t}^{*}$ : (a)-(b) deterministic model and (c)-(d) stochastic model with $(\bar{a}, \bar{k})=(0.5,2.5)$.

The stabilizing role of the market maker on the market price in both the deterministic and stochastic models is further illustrated in Figs 5 and 6 for two sets of values of $(\bar{a}, \bar{k})$ with $\alpha \in(0,1)$. The deviation of the market price from the fundamental price is measured at both mean and standard deviation levels. In the first case when $(\bar{a}, \bar{k})=(0.5,2.5)$, for the deterministic model, the market becomes unstable as $\alpha$ decreases, demonstrated by large mean and standard deviation levels as $\alpha$ decreases in Figs 5 (a) and (b). For the corresponding stochastic model, Figs 5 (c) and (d) demonstrate a similar feature. This indicates that, when the trend followers are less active and the fundamentalists are relatively more active, an increase in $\alpha$ stabilizes the market price to the fundamental price, indicating the stabilizing role of the market maker as he/she becomes more active. However, the stabilizing role of the market maker does not hold in the second case of 
$(\bar{a}, \bar{k})=(1.13,1.5)$ when the trend followers become more active and the fundamentalists are relatively less active, as illustrated in Fig. 6. In this case, for the deterministic model, the market price is stable only when the value of $\alpha$ is away from 0 and 1, see Figs 6 (a) and (b). The similar feature displays for the corresponding stochastic model.

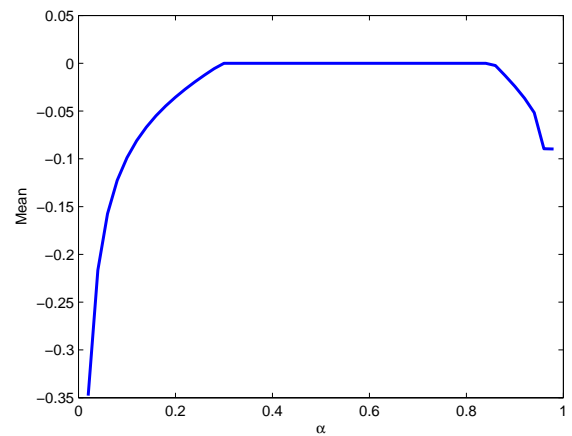

(a) Mean $\left(\sigma_{\epsilon}, \sigma_{\delta}\right)=(0,0)$

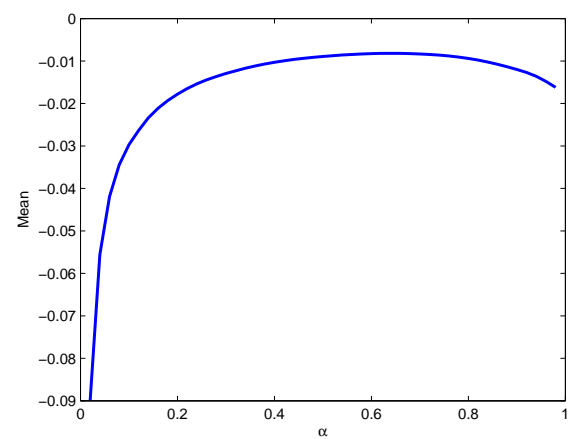

(c) Mean $\left(\sigma_{\epsilon}, \sigma_{\delta}\right)=(0.01,0.005)$

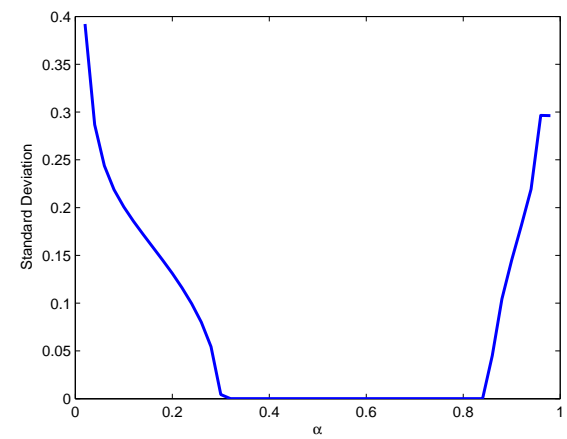

(b) $S D_{P P^{*}}\left(\sigma_{\epsilon}, \sigma_{\delta}\right)=(0,0)$

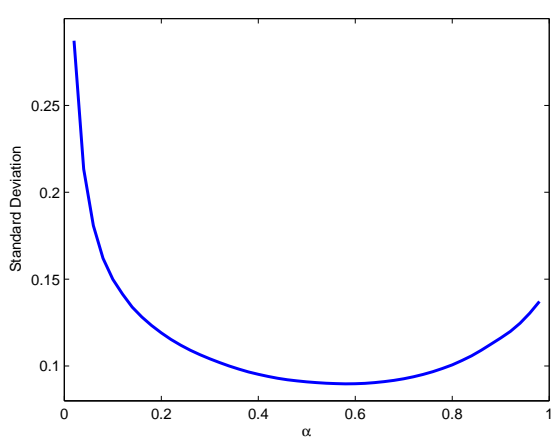

(d) $S D_{P P^{*}}\left(\sigma_{\epsilon}, \sigma_{\delta}\right)=(0.01,0.005)$

Figure 6: Mean of $P_{t}-P_{t}^{*}$ and standard deviation $S D_{P P^{*}}$ : (a)-(b) deterministic model and $(\mathrm{c})-(\mathrm{d})$ stochastic model with $(\bar{a}, \bar{k})=(1.13,1.5)$.

The above analysis demonstrates that the stabilizing role of the market maker is rather subtle and depends on the activities of the outside customers. However, when the market becomes unstable, there is a common feature shared in both cases. It is the negative deviation of the market price from the fundamental price, demonstrated by the negative mean values in Figs 5 and 6 . The negative mean values are underlined by the asymmetric belief of the trend followers which is characterized by the parameter $b$. For given $b=-1$, this represents a bearish belief of the trend followers about the future market prices. To see more clearly the impact of the bearish belief of the trend followers on the market price and the role of the noises processes, we consider two different parameter sets 
$(\alpha, \bar{a}, \bar{k})=(0.83,1.13,2.5),(0.83,1.7,2.5)$ with two sets of noises for the fundamental price and the market noise $\left(\sigma_{\epsilon}, \sigma_{\delta}\right)=(0.01,0.005),(0.01,0.5)$. We plot the time series of the price and the corresponding phase plot in price and inventory space in the deterministic cases (when $\left.\left(\sigma_{\epsilon}, \sigma_{\delta}\right)=(0,0)\right)$ and the time series of the price with two combinations of noises in Figs 7 and 8 for the two cases.

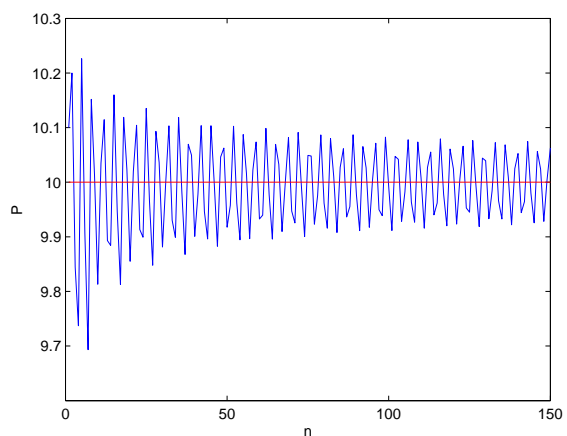

(a) Time series $\left(\sigma_{\epsilon}, \sigma_{\delta}\right)=(0,0)$

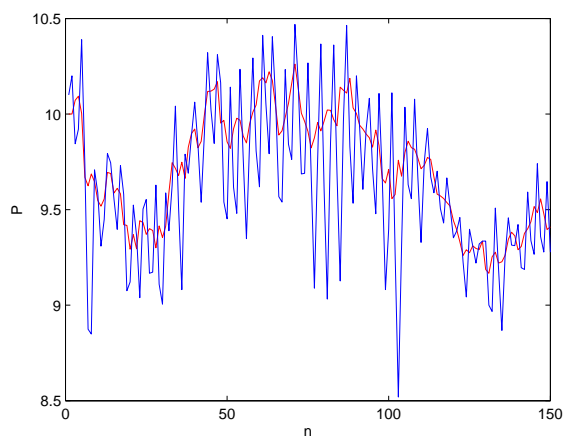

(c) Time series $\left(\sigma_{\epsilon}, \sigma_{\delta}\right)=(0.01,0.005)$

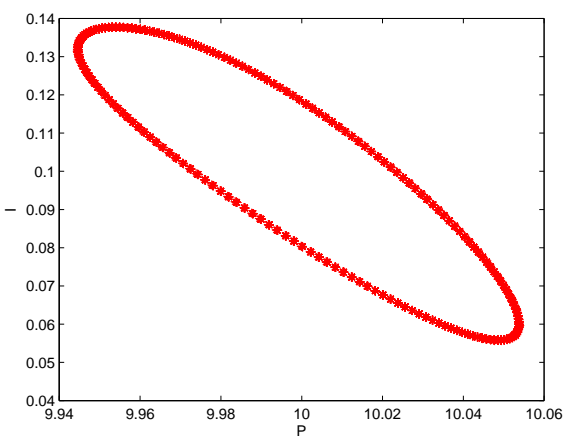

(b) Phase diagram $\left(\sigma_{\epsilon}, \sigma_{\delta}\right)=(0,0)$

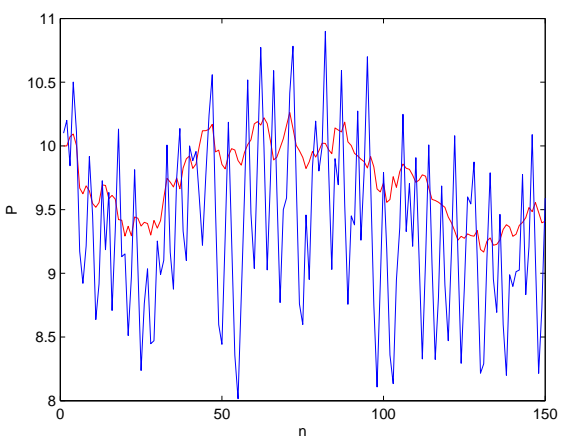

(d) Time series $\left(\sigma_{\epsilon}, \sigma_{\delta}\right)=(0.01,0.5)$

Figure 7: (a), (c), (d): Time series of fundamental price (red) and market price for the parameters indicated; (b) phase diagram of market price and market maker inventory of the deterministic model, where $\alpha=0.83, \bar{a}=1.13, \bar{k}=2.5$.

In the first case $(\alpha, \bar{a}, \bar{k})=(0.83,1.13,2.5)$, the relative reaction of the trend followers is not very strong. Based on the stability region plot in Fig. 3 (b), we know that the fundamental equilibrium is unstable for this case via a Neimark-Sacker bifurcation, leading the market price and inventory to oscillate around the fundamental equilibrium aperiodically, characterized by the quasi-periodic time series in Fig. 7 (a) and the closed cycle of the phase plot in Fig. 7 (b). When the fundamental and market noises are present, the downward tendency that is driven by the bearish beliefs of the trend followers becomes more significant, generating large deviation of the market prices from the fundamental 
prices and high volatility. In the second case $(\alpha, \bar{a}, \bar{k})=(0.83,1.7,2.5)$ when the activity of the trend followers becomes stronger (so that $\bar{a}$ increases from 1.13 to 1.7), the market displays very complicated dynamics characterized by irregular time series in Fig. 8 (a) and phase plot with a strange attractor in Fig. 8 (b), where the corresponding parameters are located far away from the Neimark-Sacker bifurcation boundary of the local stability region in Fig. 3 (c). Similarly, when the fundamental and market noises are present, the downward tendency that is driven by the trend followers becomes even more significant, generating large deviation of the market prices from the fundamental prices and high volatility. This analysis illustrates the significant impact of the asymmetric beliefs of the trend followers on the market price movement and volatility.

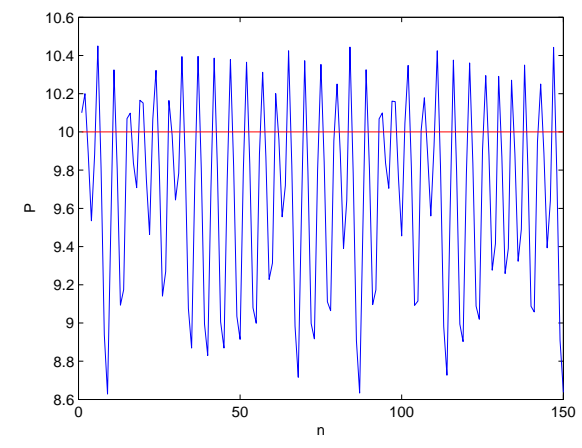

(a) Time series $\left(\sigma_{\epsilon}, \sigma_{\delta}\right)=(0,0)$

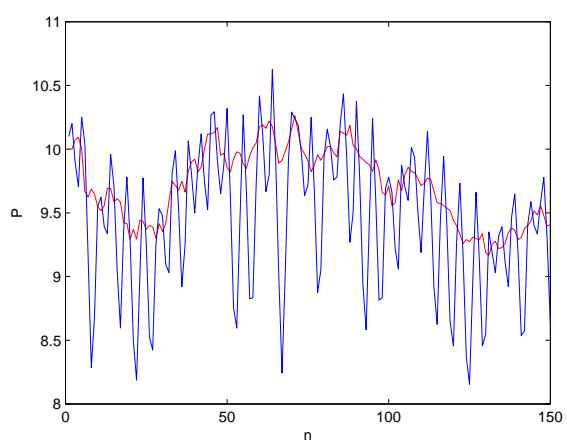

(c) Time series $\left(\sigma_{\epsilon}, \sigma_{\delta}\right)=(0.01,0.005)$

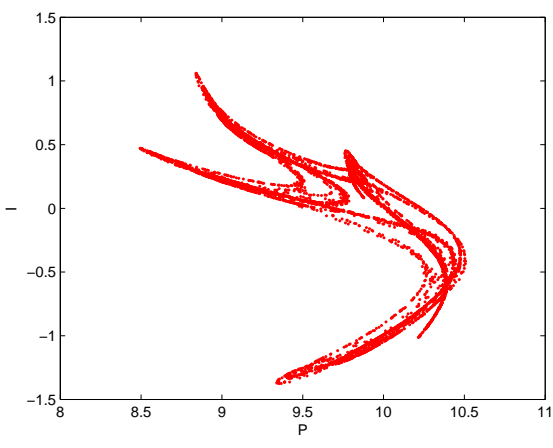

(b) Phase diagram $\left(\sigma_{\epsilon}, \sigma_{\delta}\right)=(0,0)$

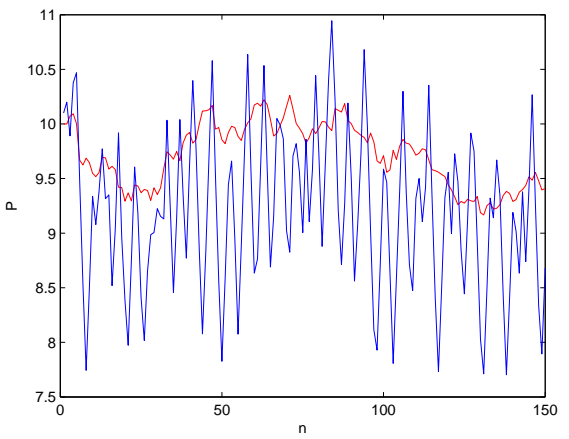

(d) Time series $\left(\sigma_{\epsilon}, \sigma_{\delta}\right)=(0.01,0.5)$

Figure 8: (a), (c), (d): Time series of fundamental price (red) and market price; (b) phase diagram of market price and market maker inventory of the deterministic model, where $\alpha=0.83, \bar{a}=1.7, \bar{k}=2.5$.

The Effects of $\alpha$ on the Average Profit of the Market Maker-As a dealer, the market maker has the obligation of maintaining a stable and orderly market. As an active investor, the market maker would like to maximize his/her profit. The question 
is: are the two roles of the market maker consistent with each other? In other words, in an effort to maximize his/her profit, is it possible that the market maker may prefer an unstable rather than a stable market? In order to answer these questions, we explore the relationship between the profits of the market maker and the speed of market maker inventory adjustment $\alpha$.

Similar to Gu (1995) and Day (1997), the profits of the market maker are assumed to be made up of a positive revenue, a variable cost, and a fixed cost. The positive revenue comes from commissions ${ }^{5}$. The variable cost consists of the loss he might suffer in carrying inventories. The fixed cost includes any fees he/she pays to maintain a seat on the exchange. Thus the profit function of the market maker is expressed as

$$
\omega_{\alpha}(t)=\psi P_{t} \pi_{t}+\left(P_{t}+D_{t}-(1+r) P_{t-1}\right) I_{t}-F
$$

where $\psi$ is the coefficient capturing commissions, $\pi_{t} \equiv n_{1}\left|z_{1, t}^{*}-z_{1, t-1}^{*}\right|+n_{2}\left|z_{2, t}^{*}-z_{2, t-1}^{*}\right|+$ $\left|\widetilde{\delta}_{t+1}-\widetilde{\delta}_{t}\right|$ is the number of shares traded in period $t, P_{t}+D_{t}-(1+r) P_{t-1}$ is the capital return per share in period $t$, and $F$ is the fixed cost. Here for convenience of analysis, and without loss of generality, we take $F=0$. For given $\alpha$, the average profit over $T$ periods is

$$
\varphi_{\alpha}(T)=\varphi_{\alpha, 1}(T)+\varphi_{\alpha, 2}(T)=\frac{1}{T} \sum_{t=1}^{T} \omega_{\alpha}(t)
$$

where

$$
\varphi_{\alpha, 1}(T)=\frac{1}{T} \sum_{t=1}^{T} \psi P_{t} \pi_{t}, \quad \varphi_{\alpha, 2}(T)=\frac{1}{T} \sum_{t=1}^{T}\left(P_{t}+D_{t}-(1+r) P_{t-1}\right) I_{t} .
$$

The following analysis of the profit focuses on the case $(\bar{a}, \bar{k})=(0.5,2.5) \operatorname{shown}^{6}$ in Fig. 9. For other cases we have similar results. In this case, Fig. 5 (d) indicates that the deviation of the market price from the fundamental price increases as $\alpha$ decreases. Correspondingly the trading becomes more active. As shown in Fig. 9 (a), the average profit of the market maker from commissions $\varphi_{\alpha, 1}$ increases as $\alpha$ decreases. However, Fig. 9 (b) reveals that the market maker suffers a loss in carrying inventory on average. But

\footnotetext{
${ }^{5}$ The commissions can also include the bid-ask spread if we take into account the more general fees and assume that the bid-ask spread is constant, as in $\mathrm{Gu}(1995)$.

${ }^{6}$ Here we take $z_{s}=0.01, I^{d}=0.001$ in order to make the mean of dividends much smaller. $D_{t} \sim$ $U(0,0.022)$ is uniformly distributed on the interval $[0,0.022]$ with a mean of $\bar{D}=0.011$. In fact, on a daily basis, the dividends effect is very small, indeed our numerous simulations reveal that the dividends hardly effect the average profits.
} 


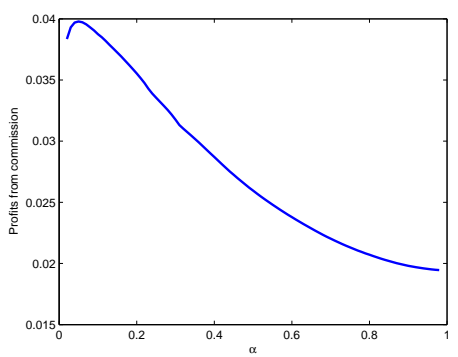

(a) $\varphi_{\alpha, 1}$

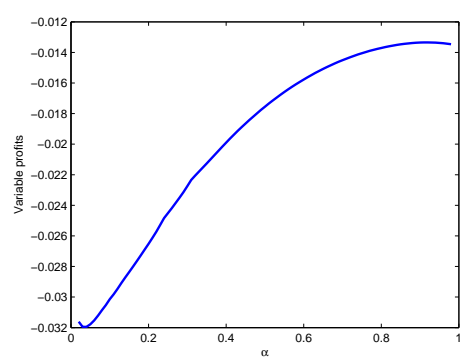

(b) $\varphi_{\alpha, 2}$

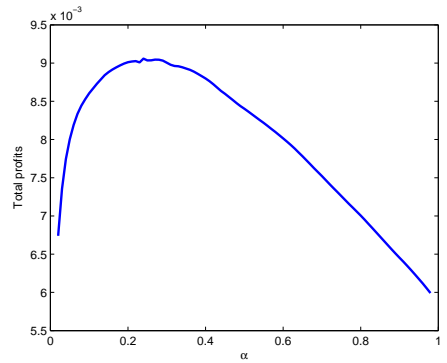

(c) $\varphi_{\alpha}$

Figure 9: (a)-(b) The average profit of the two parts; (c) the total average profits, where $(\bar{a}, \bar{k})=(0.5,2.5), \psi=0.014, z_{s}=0.01, I^{d}=0.001,\left(\sigma_{\epsilon}, \sigma_{\delta}\right)=(0.01,0.005)$ and other parameters are the same as Table 1.

the loss becomes smaller as $\alpha$ increases. The total average profit $\varphi_{\alpha}$ displayed in Fig. 9 (c) shows that, with $\psi=0.014$ (a commission fee of about $1.4 \%$ ), the profit $\varphi_{\alpha}$ of the market maker reaches its maximum for $\alpha$ about 0.2. From Figs 5 (b) and (d), we know that, for $\alpha$ about 0.2 , the market price is unstable with relatively large deviation from the fundamental price. This implies that it is possible that the market maker prefers an unstable market in order to maximize his/her own profits. A similar result is also obtained in $\mathrm{Gu}$ (1995) who studies the effects of the price adjustment speed of the market maker on his/her average profit without modelling the market maker inventory.

In summary, the impact of the market maker on the market is rather subtle, depending on the behaviour of other market participants. The asymmetric beliefs of the trend followers has a significant impact on the market price. Also, there is a conflict in the roles of the market maker as both a dealers and an active investor. This is to say that the maker maker may have an incentive to destabilize the market if that helps his/her own profit situation.

\section{Statistical Analysis of the Stochastic Model}

In this section we carry out a statistical analysis of the stochastic version of the model given by (2.16) for $\alpha=0.2$ and 0.4 . The analysis focuses on the time series properties of the market price and inventory when the fundamental equilibrium of the underlying deterministic model is stable. In particular, it would be very interesting to know if the model is able to generate the stylized facts of returns, the empirical patterns concerning 


\begin{tabular}{|c|c|c|c|c|c|c|c|c|c|c|c|c|c|}
\hline$P^{*}$ & $k^{\prime}$ & $a^{\prime}$ & $b$ & $c$ & $\mu$ & $\rho$ & $n_{1}$ & $n_{2}$ & $z_{s}$ & $I^{d}$ & $r$ & $\bar{D}$ & $a \sigma^{2}$ \\
\hline 10 & 0.38 & 0.2 & -0.8 & 0.16 & 2 & 0.1 & 0.5 & 0.5 & 1 & 0.1 & 0.0002 & 0.902 & 1 \\
\hline
\end{tabular}

Table 3: The common parameter values for the simulations of the stochastic model.

inventory (see Hasbrouck and Sofianos (1993)) and its relation to returns observed in financial markets that we discussed in the introductory section.

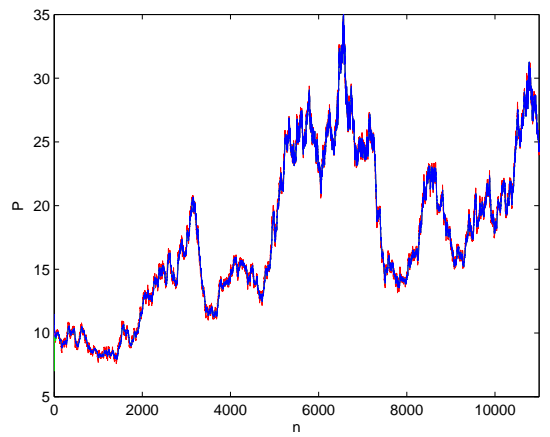

(a) Prices

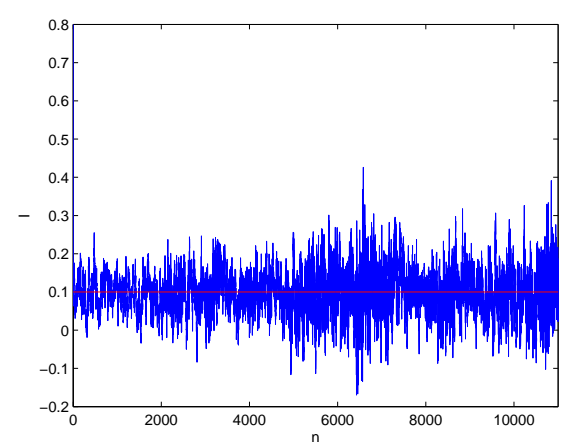

(c) Inventories

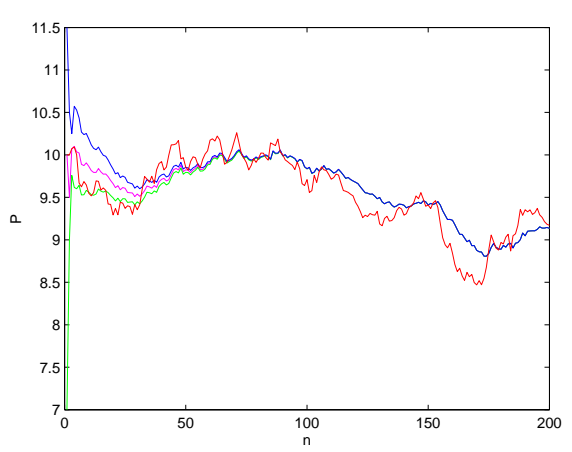

(b) Prices

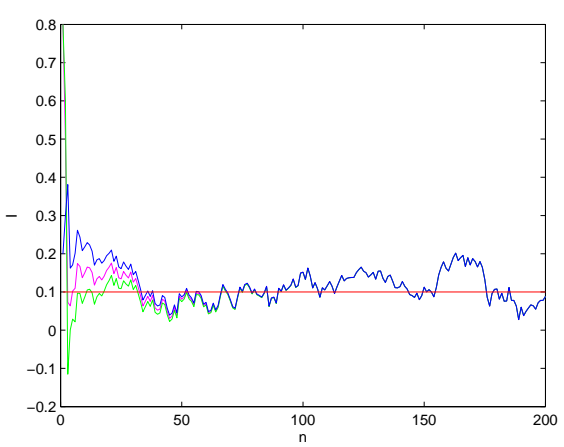

(d) Inventories

Figure 10: The time series of (a) the fundamental prices (red) and market prices, (c) the target inventory (red) and market inventories, with $T=11,000$; and time series of (b) the fundamental prices (red) and market prices, (d) the target inventory (red) and market inventories, with $T=200$ and three sets of different initial values, where $\alpha=0.2$.

For the selected parameters in Table 3 , it can be verified that for any $\alpha \in(0,1]$ the underlying deterministic model has a stable fundamental equilibrium in both price and market inventory. Consider first a special case $\alpha=0.2$. Given noisy perturbations to the fundamental price with $\sigma_{\epsilon}=0.01$ and from noise traders with $\sigma_{\delta}=0.005$ and $\alpha=0.2$, Fig. 10 plots the time series of the fundamental prices (Fig. 10 (a)) and target inventory (Fig. $10(\mathrm{c})$ ) in the red curves and the market prices (Fig 10. (a)) and 
inventories (Fig. $10(\mathrm{c})$ ) with three sets of different initial values $(P(1), I(1), P(0))=$ $(9.5,0.5,10),(10.5,0.3,10.5),(4.5,0.3,6.5)$ for $T=11,000$ time steps. These figures show that the price and inventory fluctuate around the fundamental price and the target inventory level, as has been shown in Hasbrouck and Sofianos (1993) and Hendershott and Seasholes (2007). In order to see the asymptotic behavior of the model in the long run, we choose the three sets of different initial values but the same realizations of the noise processes and plot the time series for the first 200 steps in Figs 10 (b) and 10 (d). These latter figures demonstrate the path-wise convergence of the time series with different initial values to the same random path, which implies the existence of random fixed points (see Arnold (1998)) in both price and market inventory. The connection between the dynamics of the deterministic and random models is a rather complicated issue and is beyond the scope of this paper and we refer the reader to $\mathrm{He}$ and $\mathrm{Li}$ (2008) for related discussion.

Next we examine the statistical properties of the returns calculated by $r_{t}=P_{t} / P_{t-1}-1$ and inventories (and the relationship between them) produced by the time series in Fig. 10. The time series of returns in Fig. 11 (a) displays the volatility clustering observed in financial markets. Compared with the corresponding normal distribution, the return distribution in Fig. 11 (b) shows a high degree of kurtosis and fat tails. Furthermore the plots of autocorrelations (ACs) of returns, absolute returns and squared returns in Figs 11 (c-e) show (almost) insignificant higher-order ACs but with significant ACs for lags up to 7 for the raw returns ${ }^{7}$, but significant $\mathrm{AC}$ patterns for the absolute and squared returns across long lags. This demonstrates that the model is able to generate some of the stylized facts and long-rang dependence in return volatility observed in financial markets, as mentioned by Mantegna and Stanley (2000) and Lux and Ausloos (2002). We also calculate the ACs of inventories in Fig. 11 (f), showing a positive decaying pattern with lags up to 50. This result is consistent with the empirical findings in Hasbrouck and Sofianos (1993) who find that inventories of many stocks are positively autocorrelated with lags of about 10-50 days.

Further, we use the stochastic model to examine the relationship between returns and inventories, which is shown in the second row of Table 4. We calculate the correlation coefficients of $r_{t}$ and $I_{t}-I_{t-1}, r_{t}$ and $I_{t}, I_{t}$ and $r_{t+1}$, and $r_{t-1}$ and $I_{t}$, respectively. We find that the returns are negatively related to the contemporaneous (changes of) inventories.

\footnotetext{
${ }^{7}$ If desired, the low-order ACs could be driven down to insignificant level by increasing the size of noise traders. However, this will make high kurtosis less obvious.
} 
In addition, inventories have a positive impact on the returns of the next period, while the past high returns may lead to low current inventories. Our results are consistent with the empirical findings of Hendershott and Seasholes (2007), who use 11-year inventory data from the New York Stock Exchange to empirically explore the relationship between returns and inventories. This indicates that our stochastic model is capable of generating theoretical support for the empirical findings related to the inventory and its relation to the returns. Similar to He and Li (2007), we could conduct Monte Carlo simulations and various statistical tests in order to check the robustness of these results, but we leave this task to future research.

The Effects of $\alpha$ on the Statistical Properties - To demonstrate the robustness of the statistical results reported above for changing $\alpha$, we first conduct the same analysis by replacing $\alpha$ with $\alpha=0.4$. In this case, we can obtain the same time series feature shown in Fig. 10. Fig. 12 displays the statistical properties of returns and inventories. Comparing with Fig. 11, we observe that, as $\alpha$ increase, the lags of the significant ACs of returns and inventories decrease. Especially, the lags of significant ACs for inventories decreases from 55 to 15 . This implies that the adjustment speed of the market maker inventory has an important impact on the autocorrelation of inventory. A lower speed of inventory adjustment tends to generate significant ACs for market maker inventory over longer lags. Fig. 12 also shows that in the case that both the fundamentalists and trend followers are less active so that the deterministic model is stable, the behavior of the market maker has a relatively small effect on the statistical properties.

In Table 4, we present the various correlations between returns and inventories for $\alpha=0.1,0.3$ and 0.4 . It clearly shows that as $\alpha$ increases, the correlations between returns and contemporary changes of inventories and between current inventory and future returns become weaker, but the correlations between returns and contemporary inventories and between past returns and the current inventories become stronger. We give an intuitive explanation of these changes. For the correlations between returns and contemporary changes of inventories, as $\alpha$ increases, the effect of $\alpha\left(I^{d}-I_{t}\right)$ on the desired supply of the market maker $I_{t}-I_{t+1}+\alpha\left(I^{d}-I_{t}\right)$ becomes large and hence the negative correlation between $r_{t+1}$ and $I_{t+1}-I_{t}$ decreases. Furthermore, as $\alpha$ increases, the effect of $(1-\alpha) I_{t}$ on the desired supply of the market maker $-I_{t+1}+(1-\alpha) I_{t}+\alpha I^{d}$ decreases. Thus the negative correlation between $r_{t+1}$ and $I_{t+1}$ becomes large but the positive correlation between $r_{t+1}$ and $I_{t}$ becomes small. In addition, following the same calculation in Section 3 , we can obtain the results that $S D_{P P^{*}}=0.3354$ for $\alpha=0.2$ and $S D_{P P^{*}}=0.2531$ for 


\begin{tabular}{|c|c|c|c|c|}
\hline$\alpha$ & $\operatorname{correl}\left(r_{t}, I_{t}-I_{t-1}\right)$ & $\operatorname{correl}\left(r_{t}, I_{t}\right)$ & $\operatorname{correl}\left(I_{t}, r_{t+1}\right)$ & $\operatorname{correl}\left(r_{t-1}, I_{t}\right)$ \\
\hline 0.1 & -0.9854 & -0.4556 & 0.1388 & -0.2682 \\
\hline 0.2 & -0.9659 & -0.6113 & 0.1349 & -0.3641 \\
\hline 0.3 & -0.9378 & -0.7178 & 0.0920 & -0.4315 \\
\hline 0.4 & -0.9020 & -0.8008 & 0.0360 & -0.4748 \\
\hline
\end{tabular}

Table 4: Correlation coefficients for four values of $\alpha$.

$\alpha=0.4$. That is, the deviation between the market prices and the fundamental prices for $\alpha=0.4$ is smaller than that for $\alpha=0.2$. This indicates that as $\alpha$ increases from 0.2 to 0.4 , the demand of the fundamentalists decreases and hence the effect of $n_{1} z_{1, t}^{*}$ on the inventory $I_{t+1}$ decreases and the relative effect of $n_{2} z_{2, t}^{*}$ on the inventory $I_{t+1}$ increases, where $n_{2} z_{2, t}^{*}$ is positively related to $r_{t}$. Hence the negative correlation between $r_{t}$ and $I_{t+1}$ increases.

In a word, our model can replicate most of the stylized facts in financial markets. Furthermore, as the parameter $\alpha$ changes in a certain range, the statistical properties can basically remain, except that the (auto)correlations coefficients of returns and inventories undergo some changes.

\section{Conclusion}

In this paper we develop a heterogeneous agent model of asset price and inventory in a market where price clearing is via a market maker mechanism. In setting the price each period the market maker takes account not only of the excess demand of the outside agents, who are modelled using the fundamentalist and chartist approach of the boundedly rational heterogeneous agent model literature, but also his/her inventory position, for which the market maker has some desired target level. Noise enters into the model through the fundamental price and market noise traders.

We find that the stabilizing role of the market maker can be very subtle and depends upon different levels of activity of the fundamentalists and trend followers. When the trend followers are relatively less active comparing with the fundamentalists, the market maker, as an active investor, can stabilize the market (when $\alpha$ increases). However, the stabilizing role of the market maker does not hold when the trend followers becomes relatively more active comparing with the fundamentalists. We also find that the asymmetric beliefs of the trend followers have a great impact on the market price deviation from 
the fundamental price and bearish beliefs of the trend followers have more impact when the market goes down. By examining the profitability of the market maker, we found that the market maker has an incentive to destabilize the market in order to maximize the profit. Furthermore, the corresponding random model exhibits many of the stylized facts of returns and inventories observed in empirical studies. Our model can produce the stylized facts of returns including high kurtosis, fat tails, volatility clustering. power-law behaviour of returns. It can also yield the decaying positive autocorrelation of inventories and the various relations of returns and inventories consistent with empirical findings. That is, the returns are negatively related to contemporaneous (changes of) inventories. In addition, inventories have a positive impact on the returns of the next period, while the past high returns may lead to low current inventories. Furthermore, as the parameter $\alpha$ changes in a certain range, the statistical properties can basically remain. The analysis and results obtained in this paper improve our understanding of the role of the market maker in setting the market price and his/her impact on market price volatility. Such an understanding is crucial to practical issues concerning market design, regulations and stability.

In future research, it would be of interest to build up a forecasting model on future inventory imbalances for the market maker, by which the market maker determines the parameters of the price and inventory adjustment.

\section{References}

[1] Arnold, L., 1998. Random Dynamical Systems, Springer-Verlag, Berlin.

[2] Beja, A. and M.B. Goldman, 1980. On the dynamic behavior of prices in disequilibrium. The Journal of Finance, 35, 235-247.

[3] Bradfield, J, 1979. A formal dynamic model of market making. The Journal of Financial and Quantitative Analysis, 14(2), 275-291.

[4] Brock, W.A., Hommes, C.H., 1997. A rational route to randomness. Econometrica, 65, 1059-1095.

[5] Brock, W., Hommes, C., 1998. Heterogeneous beliefs and routes to chaos in a simple asset pricing model. Journal of Economic Dynamics and Control, 22, 1235-1274. 
[6] Chen, S.H., Yeh, C.H., 2001. Evolving Traders and the business school with genetic programming: A new architecture of the agent-based artificial atock market. Journal of Economic Dynamics and Control, 25, 363-394.

[7] Chen, S.H., Yeh, C.H., 2002. On the emergent properties of artificial stock markets: the efficient market hypothesis and the rational expectations hypothesis. Journal of Economic Behavior and Organization, 49(2), 217-239.

[8] Chiarella, C., 1992. The dynamics of speculative behaviour. Annals of Operations Research, 37, 101- 123.

[9] Chiarella, C., Dieci, R., Gardini, L., 2002. Speculative behaviour and complex asset price dynamics: a global analysis. Journal of Economic Behavior and Organization, 49, 173-197.

[10] Chiarella, C., Dieci, R., He, X., 2009. On the dynamic behaviour of asset prices in disequilibrium: a survey. T. Hens and K. R. Schenk-Hoppé, eds, 'Handbook of Financial Markets: Dynamics and Evolution', pp.277-344, in the series of Handbooks in Finance (W. Ziemba, eds), Elsevier.

[11] Chiarella, C., He, X., 2001. Asset price and wealth dynamics under heterogeneous expectations. Quantitative Finance, 1(5), 509-526.

[12] Chiarella, C., He, X., 2002. Heterogeneous beliefs, risk and learning in a simple asset pricing model. Computational Economics, 19, 95-132.

[13] Chiarella, C., He, X., 2003. Heterogeneous beliefs, risk and learning in a simple asset pricing model with a market maker. Macroeconomic Dynamics, 7, 503-536.

[14] Chiarella, C., He, X., Wang, D. and Zhu, M., 2008. Stock price and market maker inventory dynamics with heterogeneous beliefs. Working Paper, School of Finance and Economics, University of Technology, Sydney.

[15] Cont, R., Bouchaud, J.P., 2000. Herd behaviour and aggregate fluctuations in financial markets. Macroeconomic Dynamics, 4, 170-196.

[16] Day, R.H., 1997. Complex dynamics, market mediation and stock price behaviour. North American Actuarial Journal, 1, 6-23. 
[17] Day, R.H., Huang, W., 1990. Bulls, bears and market sheep. Journal of Economic Behavior \& Organization, 14(3), 299-329.

[18] Farmer, J.D., Joshi, S., 2002. The price dynamics of common trading strategies. Journal of Economic Behavior $\& 5$ Organization, 49, 149-171.

[19] Franke, R., Asada, T., 2008. Incorporating positions into asset pricing models with order-based strategies. Journal of Economic Interaction and Coordination, 3, 201-227.

[20] Grossman, S.J., Miller, M.H., 1988. Liquidity and market structure. The Journal of Finance, 43(3), 617-633.

[21] Gu, M., (1995). Market mediating behaviour: an economic analysis of the security exchange specialists. Journal of Economic Behavior and Organization, 27, 237-56.

[22] Hasbrouck, J., Sofianos, G., 1993. The trades of market-makers: an analysis of NYSE specialists. The Journal of Finance, 48, 1565-1593.

[23] He, X., Li, Y., 2007. Power law behaviour, heterogeneity, and trend chasing. Journal of Economic Dynamics and Control, 31, 3396-3426.

[24] He, X., Li, Y., 2008. Heterogeneity, convergence and autocorrelations. Quantitative Finance, 8, 59-79.

[25] Hendershott, T., Seasholes, M.S., (May) 2007. Market maker inventories and stock prices. American Economic Review, 97(2), 210-214.

[26] Hansch, O., Naik, N.Y., Viswanathan, S., 1998. Do Inventories Matter in Dealership Markets? Evidence from the London Stock Exchange. The Journal of Finance, 53(5), 1623-1656.

[27] Hommes, C., 2006. Heterogeneous agent models in economics and finance, in 'Handbook of Computational Economics', K.L. Judd and L. Tesfatsion, eds, Part I, Chapter 23, 1109-1186, Elsevier.

[28] Hommes, C.H., Huang, H., Wang, D., 2005. A robust rational route to randomness in a simple asset pricing model. Journal of Economic Dynamics and Control, 29, 10431072. 
[29] Iori, G., 2002. A microsimulation of traders activity in the stock market: the role of heterogeneity, agents' interactions and trade frictions. Journal of Economic Behavior and Organization, 49, 269-285.

[30] Kirman, A., 1991. Epidemics of opinion and speculative bubbles in financial markets. In Taylor, M.P., ed. 'Money and Financial Markets', 354-368, Blackwell, Cambridge.

[31] LeBaron, B., 2001. Stochastic volatility as a simple generator of apparent financial power laws and long memory. Quantitative Finance, 1, 621C631.

[32] LeBaron, B., 2002. Short-memory traders and their impact on group learning in financial markets. Proceedings of the National Academy of Science: Colloquium, 99(Supplement 3), 7201C7206.

[33] LeBaron, B., 2006. Agent-based computational finance. In Judd, K.L., and Tesfatsion, L., eds.'Handbook of Computational Economics', Part I, Chapter 24, 1187-1233, Elsevier.

[34] Lux, T., 1995. Herd behaviour, bubbles and crashes. Economic Journal, 105, 881896.

[35] Lux, T., 2009. Financial power laws: empirical evidence, models and mechanisms. In Cioffi-Revilla, C., ed. 'Power Laws in the Social Sciences: Discovering Complexity and Non-equilibrium Dynamics in the Social Universe'.

[36] Lux, T., Ausloos, M., 2002. Market fluctuations I: Scaling, multiscaling, and their possible origins. In Bunde, A., Kropp, J. and Schellnhuber, H., eds. 'Science of disaster: climate disruptions, heart attacks, and market crashes'. Springer, Berlin,

[37] Madhavan, A., Smidt, S., 1993. An analysis of changes of specialist inventories and quotations. The Journal of Finance, 48(5), 1595-1628.

[38] Madhavan, A., Sofianos, G., 1998. An empirical analysis of NYSE specialist trading. Journal of Financeal Economics, 48, 189-210.

[39] Mantegna, R.N., Stanley, H.E., 2000. An introduction to econophysics: correlations and complexity in finance. Cambridge University Press, Cambridge.

[40] O’Hara, M., 1995. Market Microstructure Theory. Blackwell, USA. 
[41] Sethi, R., 1996. Endogenous regime switching in speculative markets. Structural Change and Economic Dynamics, 7, 99-118.

[42] Snell, A., Tonks I., 1998. Testing for asymmetric information and inventory control effects in market maker behaviour on the London Stock Exchange. Journal of Empirical Finance, 5, 1-25.

[43] Westerhoff, F., 2003. Market-maker, inventory control and foreign exchange dynamics. Quantitative Finance, 3, 363-369.

[44] Westerhoff, F., 2004. Greed, fear and stock market dynamics. Physica A, 343, 635642.

[45] Westerhoff, F., 2008. The use of agent-based financial market models to test the effectiveness of regulatory policies. Jahrbücher für Nationalökonomie und Statistik (Journal of Economics and Statistics), 195-227.

[46] Wyss, B.O., 2001. Fundamentalists of the stock market. McGraw-Hill, New York.

[47] Zheng, M., 2007. Dynamics of asset pricing models with heterogeneous Beliefs. Doctoral dissertation at Peking University. 


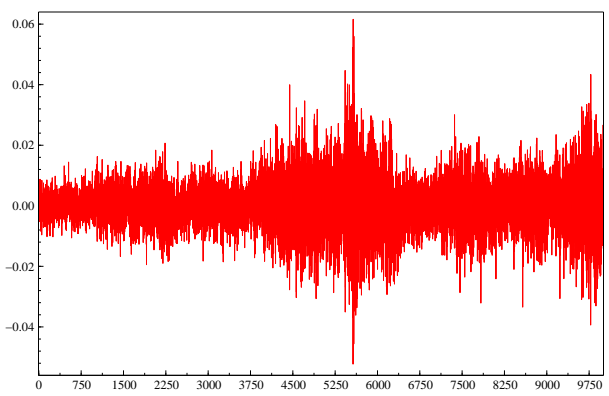

(a) Time series of the returns

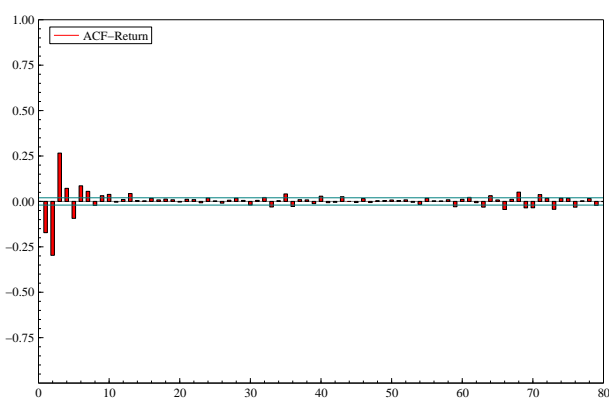

(c) Autocorrelation of the returns

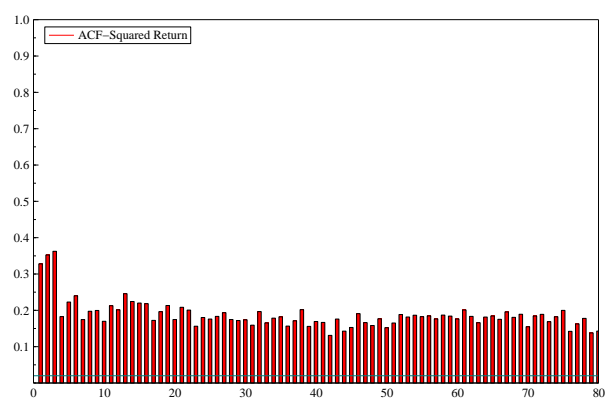

(e) Autocorrelation of the squared returns

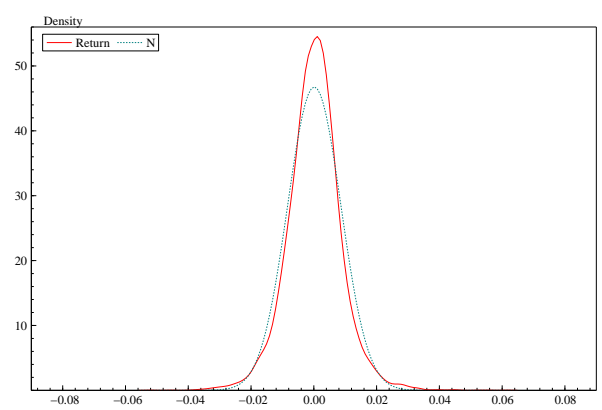

(b) Distribution of the returns

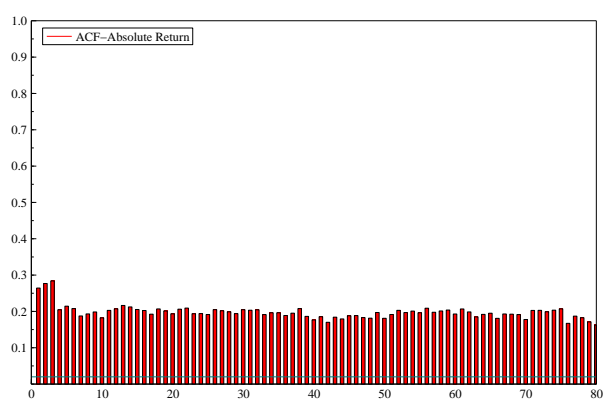

(d) Autocorrelation of the absolute returns

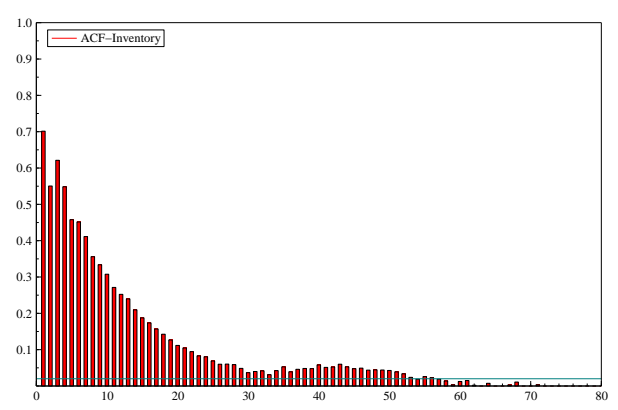

(f) Autocorrelation of the inventories

Figure 11: Time series of the returns (a), distribution of the returns (b), autocorrelations for the returns (c), the absolute returns (d) and the squared returns (e), autocorrelation of the inventories (f), where $\alpha=0.2$. 


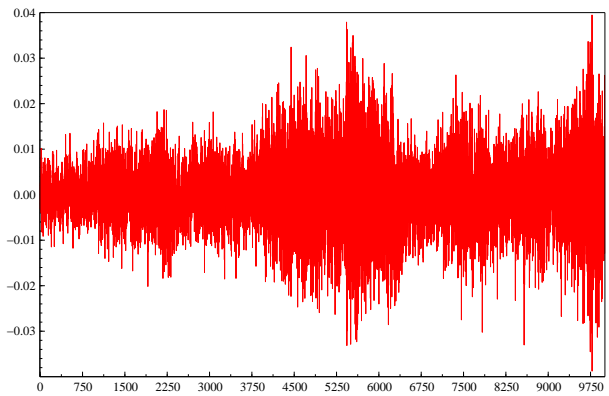

(a) Time series of the returns

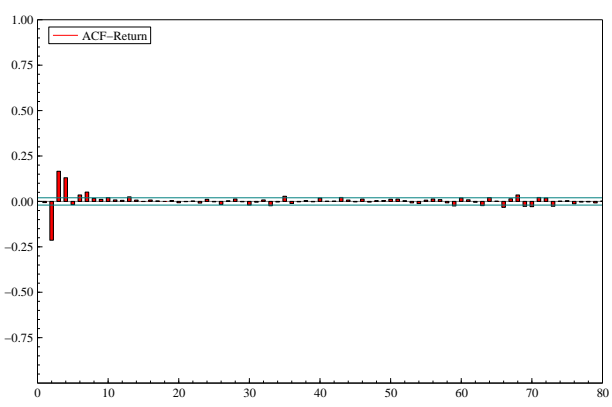

(c) Autocorrelation of the returns

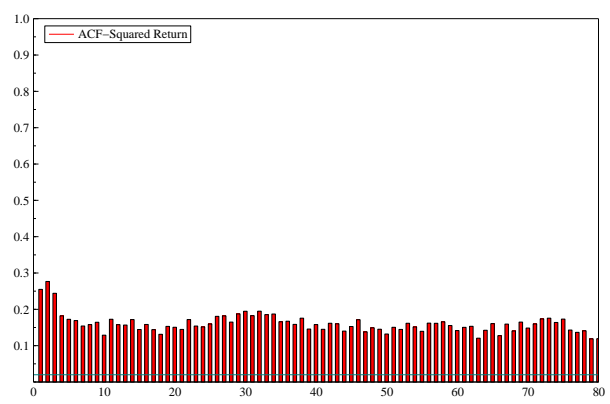

(e) Autocorrelation of the squared returns

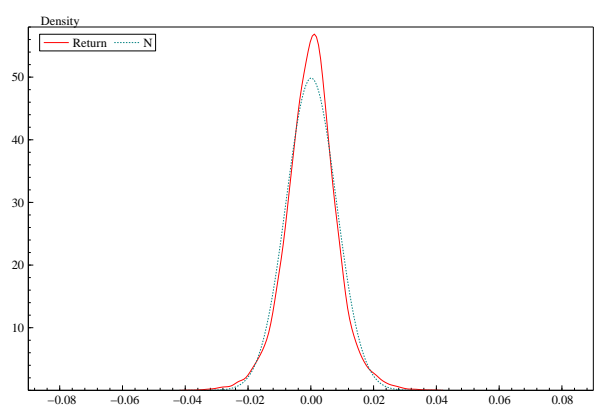

(b) Distribution of the returns

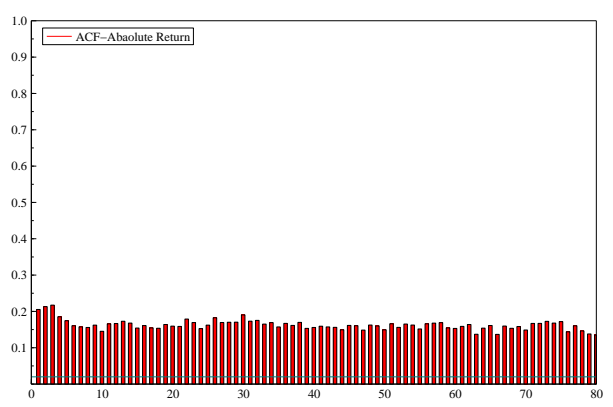

(d) Autocorrelation of the absolute returns

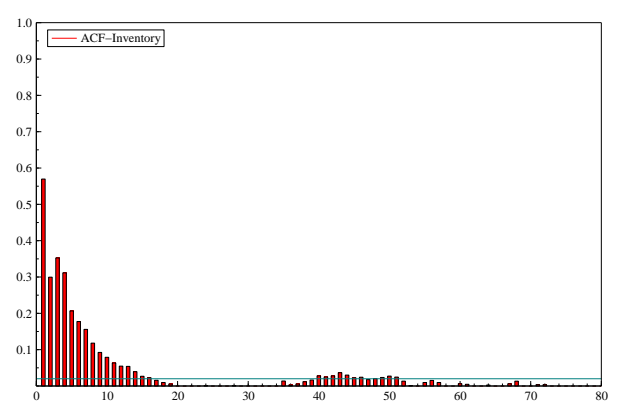

(f) Autocorrelation of the inventories

Figure 12: Time series of the returns (a), distribution of the returns (b), autocorrelations for the returns (c), the absolute returns (d) and the squared returns (e), autocorrelation of the inventories (f), where $\alpha=0.4$. 